\title{
Antagonistic activation patterns underlie multi-functionality of the right temporo-parietal junction
}

\author{
Danilo Bzdok, Robert Langner, Simon Eickhoff \\ Institute of Neuroscience and Medicine (INM-1) \\ Research Center Jülich \\ Jülich, Germany \\ d.bzdok@fz-juelich.de
}

\author{
Angela R. Laird, Peter T. Fox \\ Research Imaging Institute \\ University of Texas Health Science Center \\ San Antonio, Texas, USA
}

\begin{abstract}
The right temporo-parietal junction (RTPJ) is consistently implicated in two cognitive domains - attention and social cognitions. We conducted multi-modal connectivitybased parcellation to investigate potentially separate functional modules within RTPJ implementing this cognitive dualism. Both task-constrained meta-analytic coactivation mapping and task-free resting-state connectivity analysis independently identified two distinct clusters within RTPJ, subsequently characterized by network mapping and functional forward/reverse inference. The anterior cluster increased activity concomitantly with a midcingulate-motor-insular network, functionally associated with attention, and decreased activity with a parietal network, functionally associated with social cognition and introspection. The posterior cluster showed the exactly opposite association pattern. Our data thus suggest that RTPJ links two antagonistic brain networks processing external versus internal information.
\end{abstract}

anti-correlation, connectivity-based parcellation, functional decoding, temporo-parietal junction

\section{INTRODUCTION}

The human temporo-parietal junction is a supramodal area at the posterior Sylvian fissure [1]. The inconsistent anatomical naming of this brain area parallels its implication in various different psychological processes, especially in the right hemisphere (RTPJ). In particular, an extensive body of work implies selectivity of the RTPJ for basic attentional processes, while a similarly extensive, yet independent body of literature claims selectivity for higherlevel processing of social information [2].

Conceivably, this apparent contradiction may be explained by functional heterogeneity within the RTPJ. This hypothesis was tested by connectivity-based parcellation (CBP), functional connectivity mapping, and quantitative functional decoding on a seed region comprising RTPJ activity associated with previously proposed functions. First, we conducted CBP to exploit the unique set of input and output connections of any particular functional cortical module [3] to "blindly" infer functional parcellations from connectivity data [4]. Second, the connectivity-derived subregions were characterized by determining their brain-wise connectivity profiles based on two complementary measures of functional connectivity - task-related meta-analytic connectivity modeling (MACM) and task-unrelated resting- state functional connectivity (RSFC). Third, we delineated the sub-regions' functional profiles from above-chance taxonomic associations with meta-data archived in the BrainMap database.

\section{METHODS}

To both accommodate lacking neuroanatomical consensus and acknowledge the diverse functions ascribed to the RTPJ, the volume of interest for CBP was constructed by merging results of three meta-analyses of neuroimaging data on sustained attention [5], sensorimotor control [6], and theory of mind [7]. The composite VOI was then submitted to a CBP procedure that grouped seed voxels as a function of their similarities in whole-brain connectivity patterns [8], independently using MACM and RSFC.

Delineation of whole-brain MACM maps for each voxel of the RTPJ seed region was performed based on the BrainMap database [9]. We constrained our analysis to archived functional magnetic resonance imaging (fMRI) and positron emission tomography (PET) experiments from "normal mapping" studies in healthy participants that report results as coordinates in stereotaxic space. These inclusion criteria yielded $\sim 6,500$ eligible experiments. For each seed voxel we identified those among the BrainMap experiments that reported activation foci at or in the immediate vicinity of that seed voxel. A challenge in constructing coactivation maps is the limited number of experiments activating precisely at a particular seed voxel. Hence, pooling across the close spatial neighborhood has become the dominant approach in MACM analysis. In the present study, we realized such pooling across a closely adjacent neighborhood, as needed to reliably determine the coactivation patterns of a given seed voxel, by identifying those BrainMap experiments that reported closest activation to that voxel. That is, the experiments associated with each seed voxel were defined by activation at or in the immediate vicinity of this specific seed voxel. The ensuing experiment set was then submitted to quantitative activation likelihood estimation (ALE) meta-analysis [10] to yield co-activation maps for the current seed voxel. Each seed voxels' coactivation map thus indicates how likely voxels/areas throughout the brain are to increase metabolic activity concomitantly with that seed voxel. In sum, this approach 
allowed for a robust and unbiased definition of coactivation patterns in spite of the variable and often rather low number of foci at each particular voxel.

Seed-voxel-wise whole-brain connectivity was likewise assessed using resting-state correlations as an independent modality of functional connectivity for cross-validation across different brain states. RSFC fMRI images were acquired in 100 healthy volunteers (50 female, mean age 45.2 years) without any record of neurological or psychiatric disorders. All participants gave written informed consent prior to entering the study. Prior to the imaging session, participants were instructed to keep their eyes closed and just let their mind wander without thinking of anything in particular. For each participant, 300 RSFC EPI images were acquired using blood-oxygen-level-dependent (BOLD) contrast [gradient-echo EPI pulse sequence, $\mathrm{TR}=2.2 \mathrm{~s}, \mathrm{TE}=$ $30 \mathrm{~ms}$, flip angle $=90^{\circ}$, in-plane resolution $=3.1 \times 3.1 \mathrm{~mm}^{2}$, 36 axial slices (3.1 mm thickness) covering the entire brain]. The first four scans were discarded as dummy images. Further processing relied on SPM8 (www.fil.ion.ucl.ac.uk/spm). The mean EPI image for each participant was then spatially normalized to the MNI single subject template using the 'unified segmentation' approach [11]. Finally, images were smoothed by a 5-mm FWHM Gaussian kernel.

The time-series RSFC data of each individual seed voxel were processed as follows: First, nuisance variables were removed (the six motion parameters, the first derivative of the realignment parameters, mean gray matter, white matter, and CSF signal per time-point, and coherent signal changes across the whole brain as reflected by the first five components of a principal component analysis (PCA) decomposition of the whole-brain time-series). Second, all of these nuisance variables entered the model as first-order and - except for the PCA components - also as second-order terms. Third, data were then band-pass filtered preserving frequencies between 0.01 and $0.08 \mathrm{~Hz}$. Fourth, we correlated the times-series of each individual seed voxel with those of any other brain voxel. Fifth, the ensuing correlation values were transformed into Fisher's Z-scores. In sum, correlations between spontaneous metabolic fluctuations throughout the brain during mind-wandering in the absence of an externally structured task allowed quantifying the connectivity strength of the current seed voxel with any other voxel.

To identify possibly distinct RTPJ sub-regions with unique connectivity patterns we performed CBP based on the seed-voxel-wise MACM and RSFC analyses. Independent for each modality, the brain-wide connectivity profiles for all seed voxels were combined into a $N_{S} \times N_{B}$ coactivation matrix, where $N_{S}$ denotes the number of seed voxels and $\mathrm{N}_{\mathrm{B}}$ the number of voxels in the reference brain volume.

The most appropriate number of clusters in RTPJ was then, analogous to previous CBP approaches [4], determined in a $\mathrm{N}_{\mathrm{S}} \times \mathrm{N}_{\mathrm{S}}$ cross-correlation matrix. This matrix reflected how strongly the connectivity profiles of each pair of seed voxels correlated with each other. In particular, this matrix was spectrally reordered to minimize the cross-correlation values off the diagonal, hereby forcing voxels whose connectivity profiles are highly correlated close to each other. In doing so, sets of seed voxels emerged that were strongly correlated with each other and weakly correlated with the rest of the matrix. It was this spectrally reordered correlation matrix that favored parcellation into a specific number of clusters.

Further quantitative identification of these distinct clusters that feature similar brain-wide coactivation profiles was performed by hierarchical cluster analysis. In this approach, individual voxels initially form separate clusters which are then successively included into a growing hierarchy by merging the most similar clusters into progressively larger sets of voxels. Correlation between the brain-wide connectivity profiles of seed voxels was used as a similarity measure and average linkage criterion for cluster merging. The individual seed voxels were thus merged depending on the correspondence of their connectivity profiles to identify clusters within the VOI that feature similar functional connectivity.

Following parcellation of the seed region based on regional heterogeneity in functional connectivity, additional MACM and RSFC analyses were performed on each of the ensuing clusters to characterize their whole-brain connectivity patterns. It is important to note that the above MACM and RSFC analyses assessed seed-voxel-wise connectivity patterns of individual seed voxels, while we here assessed the overall connectivity pattern of a set of seed voxels, i.e., the connectivity of the entire cluster.

To delineate areas showing task-dependent and taskindependent functional connectivity with the derived subregions in the RTPJ, we performed a conjunction analysis of the MACM and RSFC results using the strict minimum statistics [12]. In practice, regions connected with the seed in both connectivity modalities were delineated by computing the intersection of the (cluster-level family-wiseerror-corrected) connectivity maps from the two analyses detailed above.

After the first part (connectivity-derived identification of distinct clusters in the RTPJ) and second part (delineation of each clusters' convergent connectivity profile across MACM and RSFC) of our study, the clusters and their thus determined networks were individually submitted to functional profiling, as the third and last part. The functional characterization was based on the BrainMap meta-data that describe each neuroimaging experiment included in the database. Forward inference on the functional characterization then tests the probability of observing activity in a brain region given knowledge of the psychological process, whereas reverse inference tests the probability of a psychological process being present given knowledge of activation in a particular brain region. In the forward inference approach, a cluster's functional profile 
was determined by identifying taxonomic labels for which the probability of finding activation in the respective cluster was significantly higher than the overall chance (across the entire database) of finding activation in that particular cluster. Significance was established using a binomial test $(p<0.001)$. That is, we tested whether the conditional probability of activation given a particular label $[\mathrm{P}($ Activation|Task $)]$ was higher than the baseline probability of activating the region in question per se $[\mathrm{P}($ Activation $)]$. In the reverse inference approach, a cluster's functional profile was determined by identifying the most likely behavioral domains and paradigm classes given activation in a particular cluster. This likelihood $\mathrm{P}($ Task $\mid$ Activation) can be derived from $\mathrm{P}$ (Activation|Task) as well as $\mathrm{P}$ (Task) and $\mathrm{P}$ (Activation) using Bayes' rule. Significance was then assessed by means of a chi-square test $(\mathrm{p}<0.001)$.

\section{RESULTS}

Data-driven parcellation of the RTPJ yielded a robust two-cluster-solution with a 90\%-convergence across MACM and RSFC analyses. In contrast, the two CBPmodalities diverged strongly when attempting a more finegrained clustering. In the three-cluster solution, only $38 \%$ of the seed voxels were assigned congruently across both analyses. The distinction into two clusters, therefore, represents the most robust regional differentiation within RTPJ.

The ensuing anterior (aRTPJ) and posterior (pRTPJ) clusters were then fed into separate MACM and RSFC analyses. We found a double anti-correlation between the clusters as bilateral mid-cingulate cortex/supplementary motor area (MCC/SMA) and anterior insula/inferior frontal gyrus (AI/IFG) were not only positively coupled with the aRTPJ across MACM and RSFC but also negatively coupled with the pRTPJ in the RSFC analysis. Conversely, bilateral inferior parietal cortex (IPC) and precuneus were positively coupled with the pRTPJ and negatively coupled with the aRTPJ. From a neurophysiological perspective, one set of brain areas thus probably increases metabolic activity together with the aRTPJ and decreases activity with the pRTPJ, while another set of brain areas shows the opposite pattern. These findings suggest a functional anti-correlation between aRTPJ and pRTPJ.

Functional decoding using forward/reverse inference associated the aRTPJ cluster with auditory, visual, and speech discrimination tasks as well as action execution. The aRTPJ network was associated with pain perception and tactile-attentive tasks as well as action execution and motor control. In summary, functional forward and reverse inference linked aRTPJ to attentional-perceptual and actionrelated processes. In contrast, the pRTPJ cluster was associated with social-cognitive, theory-of-mind, and deception tasks as well as memory encoding and retrieval. The pRTPJ network was associated with explicit (especially episodic) memory retrieval and semantic discrimination as well as social-cognition and theory-of-mind tasks. In summary, functional forward and reverse inference linked pRTPJ to social-cognitive and memory-related processes.

\section{DICUSSION}

The functional role of the RTPJ has long remained enigmatic given implication in very heterogeneous mental functions, especially lower-order attention-/action-related cognition and higher-order social cognition. The unbiased RTPJ seed region was shown to contain two connectionally and functionally distinct modules. The complementary combination of connectivity analyses and quantitative forward/reverse functional decoding related aRTPJ and its network to supramodal stimulus-driven, external attention as well as (motor) control processes, while pRTPJ and its associated network were related to stimulus-independent, internal social-cognitive and (autobiographical) memory processes.

Forward and reverse functional decoding congruently associated the anterior RTPJ cluster with visual, auditory, and speech discrimination tasks as well as action execution, while its network was congruently associated with painful corporal stimulation and tactile-attentive tasks as well as action execution and motor control. In short, aRTPJ and the functionally connected (across MACM and RSFC) bilateral $\mathrm{MCC} / \mathrm{SMA}$, and AI/IFG were intimately related to attending to heterogeneous environmental stimuli of multiple modalities as well as controlling motor execution. In fact, attending to various stimuli and indicating responses to those by hand movement is the essence of most neuroimaging paradigms. The present functional profiling results thus predict that the aRTPJ network should increase activity in most neuroimaging studies regardless of experimental variables, such as stimuli and paradigm. Indeed, the brain areas comprising the aRTPJ network were shown to have the highest probability of activation in neuroimaging studies across all cognitive disciplines by large-scale meta-analyses of two separate datasets of more than 1000 studies each [13-14]. Regarding stimulus processing on the one hand, brain areas connected to aRTPJ further responded to all changes of visual, auditory, or tactile stimulation [15]. Regarding motor response on the other hand, neural activity in these brain areas was linked to trial-by-trial reaction time variability of the participants' responses across diverse cognitive tasks [16]. These previous findings consolidate the broad recruitment of the aRTPJ network during modality-independent external attention and motor response processes.

In contrast to aRTPJ, functional decoding congruently associated both the posterior RTPJ cluster and its network with social cognition and theory of mind, as well as memory encoding and (episodic) memory retrieval. The pRTPJ cluster was also functionally associated with deception tasks. In short, pRTPJ, functionally connected (across MACM and RSFC) to the bilateral IPC and precuneus, was intimately related to social and memory processes. On the 
one hand, prior research frequently implicated these network areas here consistently connected to the pRTPJ in higher social processes, including perspective-taking [17], social judgments [18], imagination-driven empathy [19], and moral decisions [20]. On the other hand, further research also frequently implicated the pRTPJ network in memory processes, including autobiographical/episodic memory retrieval [21] and semantic processing [22]. The present results thus tie these two largely independent literature streams and suggest a possible neural relationship between social-cognitive and episodic-memory-related processes.

Thus, aRTPJ might modulate attention to salient environmental events, while pRTPJ might be involved in memory-instructed mental imagery of hypothetical social scenes potentially guiding online behavior. Anti-correlation between these functionally antagonistic areas/networks could be a neurophysiological basis for switching between exteroception-driven and introspection-driven mind sets. Ultimately, reciprocal anti-correlation between (adjacent) cortical modules might constitute a key organizational principle of the human cerebral cortex that can explain multi-functionality.

\section{ACKNOWLEDGMENT}

This study was supported by the Human Brain Project (R01-MH074457-01A1, PTF, ARL, SBE), the Helmholtz Initiative on Systems-Biology "The Human Brain Model" (KZ, SBE), and the German National Academic Foundation (DB). The authors declare no conflict of interest.

\section{REFERENCES}

[1] M. L. Seghier, "The Angular Gyrus: Multiple Functions and Multiple Subdivisions," Neuroscientist, vol. 19, pp. 43-61, doi: $10.1177 / 1073858412440596$

[2] M. Corbetta, G. Patel, and G. L. Shulman, ,The reorienting system of the human brain: from environment to theory of mind," Neuron, vol. 58, 2008, pp. 306-324, doi: S0896-6273(08)00369-3.

[3] R. E. Passingham, K. E. Stephan, and R. Kotter, "The anatomical basis of functional localization in the cortex," Nat Rev Neurosci, vol. 3, pp. 606-16, Aug 2002, doi: 10.1038/nrn893.

[4] H. Johansen-Berg, T. E. Behrens, M. D. Robson, I. Drobnjak, M. F. Rushworth, J. M. Brady, S. M. Smith, D. J. Higham, and P. M. Matthews, "Changes in connectivity profiles define functionally distinct regions in human medial frontal cortex," Proc Natl Acad Sci U S A, vol. 101, pp. 13335-40, Sep 7 2004, doi: 10.1073/pnas.0403743101.

[5] R. Langner and S. Eickhoff, "A meta-analytic review of the neural mechanisms of vigilant attention," Psychol Bull, p. in press, 2012, doi: $10.1037 / \mathrm{a} 0030694$

[6] O. Jakobs, R. Langner, S. Caspers, C. Roski, E. C. Cieslik, K. Zilles, A. R. Laird, P. T. Fox, and S. B. Eickhoff, "Across-study and withinsubject functional connectivity of a right temporo-parietal junction subregion involved in stimulus-context integration," Neuroimage, vol. 60, pp. 2389-2398, Feb 23 2012, doi: S1053-8119(12)00215-7.

[7] D. Bzdok, L. Schilbach, K. Vogeley, K. Schneider, A. R. Laird, R. Langner, and S. B. Eickhoff, "Parsing the neural correlates of moral cognition: ALE meta-analysis on morality, theory of mind, and empathy," Brain Struct Funct, vol. 217, pp. 783-796, Jan 24 2012, doi: 10.1007/s00429-012-0380-y.
[8] S. B. Eickhoff, D. Bzdok, R. A. Laird, C. Roski, S. Caspers, K. Zilles, and P. T. Fox, "Co-activation patterns distinguish cortical modules, their connectivity and functional differentiation," Neuroimage, vol. 57, 2011, pp. 938-949, doi: 10.1016/j.neuroimage.2011.05.021.

[9] P. T. Fox and J. L. Lancaster, "Opinion: Mapping context and content: the BrainMap model," Nat Rev Neurosci, vol. 3, pp. 319-21, Apr 2002, doi: 10.1038/nrn789.

[10] S. B. Eickhoff, A. R. Laird, C. Grefkes, L. E. Wang, K. Zilles, and P. T. Fox, "Coordinate-Based Activation Likelihood Estimation MetaAnalysis of Neuroimaging Data: A Random-Effects Approach Based on Empirical Estimates of Spatial Uncertainty," Hum Brain Mapp, vol. 30, pp. 2907-2926, 2009, doi: 10.1002/hbm.20718.

[11] J. Ashburner and K. J. Friston, "Unified segmentation," Neuroimage, vol. 26, pp. 839-51, Jul 1 2005, doi: S1053-8119(05)00110-2.

[12] T. Nichols, M. Brett, J. Andersson, T. Wager, and J. B. Poline, "Valid conjunction inference with the minimum statistic," Neuroimage, vol. 25, pp. 653-60, Apr 15 2005, doi: S1053-8119(04)00750-5.

[13] S. M. Nelson, N. U. Dosenbach, A. L. Cohen, M. E. Wheeler, B. L. Schlaggar, and S. E. Petersen, "Role of the anterior insula in tasklevel control and focal attention," Brain Struct Funct, vol. 214, pp. 669-80, Jun 2010, doi: 10.1007/s00429-010-0260-2.

[14] T. Yarkoni, R. A. Poldrack, T. E. Nichols, D. C. Van Essen, and T. D. Wager, "Large-scale automated synthesis of human functional neuroimaging data," Nat Methods, vol. 8, pp. 665-70, Aug 2011, doi: 10.1038/nmeth. 1635

[15] J. Downar, A. P. Crawley, D. J. Mikulis, and K. D. Davis, "A multimodal cortical network for the detection of changes in the sensory environment," Nat Neurosci, vol. 3, pp. 277-283, 2000.

[16] T. Yarkoni, D. M. Barch, J. R. Gray, T. E. Conturo, and T. S. Braver, "BOLD correlates of trial-by-trial reaction time variability in gray and white matter: a multi-study fMRI analysis," PLoS One, vol. 4, p. e4257, 2009, doi: 10.1371/journal.pone.0004257.

[17] R. A. Mar, "The neural bases of social cognition and story comprehension," Annu Rev Psychol, vol. 62, pp. 103-34, 2011, doi: 10.1146/annurev-psych-120709-145406.

[18] J. B. Freeman, D. Schiller, N. O. Rule, and N. Ambady, "The Neural Origins of Superficial and Individuated Judgments About Ingroup and Outgroup Members," Hum Brain Mapp, vol. 31, pp. 150-159, 2010, doi: $10.1002 / \mathrm{hbm} .20852$.

[19] C. Lamm, J. Decety, and T. Singer, "Meta-analytic evidence for common and distinct neural networks associated with directly experienced pain and empathy for pain," Neuroimage, vol. 54, pp. 2492-502, Feb 1 2011, doi: S1053-8119(10)01306-6.

[20] D. Bzdok, L. Schilbach, K. Vogeley, K. Schneider, A. R. Laird, R. Langner, and S. B. Eickhoff, "Parsing the neural correlates of moral cognition: ALE meta-analysis on morality, theory of mind, and empathy," Brain Struct Funct, vol. 217, pp. 783-796, Jan 24 2012, doi: 10.1007/s00429-012-0380-y.

[21] R. N. Spreng, R. A. Mar, and A. S. Kim, "The common neural basis of autobiographical memory, prospection, navigation, theory of mind, and the default mode: a quantitative meta-analysis," J Cogn Neurosci, vol. 21, pp. 489-510, Mar 2009, doi: 10.1162/jocn.2008.21029.

[22] J. R. Binder, R. H. Desai, W. W. Graves, and L. L. Conant, "Where is the semantic system? A critical review and meta-analysis of 120 functional neuroimaging studies," Cereb Cortex, vol. 19, pp. 2767-96, Dec 2009, doi: bhp055. 